\title{
SUBGROUPS OF 3-FACTOR DIRECT PRODUCTS
}

\author{
Daniel Neuen ${ }^{1}$ - Pascal Schweitzer ${ }^{2}$ \\ ${ }^{1}$ Department of Computer Science 7, RWTH Aachen University, Aachen, GERMANY \\ ${ }^{2}$ Department of Computer Science, Technical University Kaiserslautern, Kaiserslautern, \\ GERMANY
}

\begin{abstract}
Extending Goursat's Lemma we investigate the structure of subdirect products of 3-factor direct products. We construct several examples and then provide a structure theorem showing that every such group is essentially obtained by a combination of the examples. The central observation in this structure theorem is that the dependencies among the group elements in the subdirect product that involve all three factors are of Abelian nature. In the spirit of Goursat's Lemma, for two special cases, we derive correspondence theorems between data obtained from the subgroup lattices of the three factors (as well as isomorphisms between arising factor groups) and the subdirect products. Using our results we derive an explicit formula to count the number of subdirect products of the direct product of three symmetric groups.
\end{abstract}

\section{Introduction}

The lemma of G o ursat [8] is a classic result of group theory that characterizes the subgroups of a direct product of two groups $G_{1} \times G_{2}$. A version of the lemma also provides means to describe the subgroups of $G_{1} \times G_{2}$ by inspecting the subgroup lattices of $G_{1}$ and $G_{2}$ and considering isomorphisms between arising factor groups.

In an expository article, Anderson and Camillo [1] demonstrate for example the applicability of Goursat's lemma to determine normal subgroups of $G_{1} \times G_{2}$, to count the number of subgroups of $S_{3} \times S_{3}$, and to prove the Zassenhaus Lemma. They also describe how Goursat's Lemma can be stated in the context of rings, ideals, subrings and in modules. The lemma itself can

(C) 2019 Mathematical Institute, Slovak Academy of Sciences.

2010 Mathematics Subject Classification: 20 E34.

Keyw ords: subdirect products, Goursat's Lemma, counting subgroups of products.

Funded by the Excellence Initiative of the German federal and state governments.

Licensed under the Creative Commons Attribution-NC-ND 4.0 International Public License. 
also be found in various introductory algebra and group theory texts (e.g., 9 , pp. 63-64], [11, p. 75]).

While Goursat's Lemma applies to subgroups of the direct product of two groups, in this work we are concerned with subgroups of the direct product of three groups.

It seems that there is no straightforward generalization to three factors. Indeed, B a u er, S en, and Zvengrowski 2] developed a generalization to an arbitrary finite number of factors by devising a non-symmetric version of Goursat's lemma for two factors that can then be applied recursively. A more category theory focused approach is taken by $\mathrm{Gek}$ a s [7]. However no simple correspondence theorem between the subdirect products of 3-factor direct products and data depending on the sublattice of the subgroups of the factors and isomorphisms between them is at hand. In fact, in [2] the authors exhibit two Abelian examples that stand in the way of such a correspondence theorem by sharing the various characteristic subgroups and isomorphisms between them and yet being distinct. Both these papers are able to recover several identities provided by Remak [14] who is explicitly concerned with 3-factor subdirect products.

In this paper we analyze the structure of subdirect products of 3-factor direct products. To this end we construct several examples of such groups and then provide a structure theorem showing that every such group is essentially obtained by a combination of the examples. The central observation in this structure theorem is that the dependencies among the group elements in the subdirect product that involve all three factors are of Abelian nature. We call a subdirect product of $G_{1} \times G_{2} \times G_{3}$ 2-factor injective if each of the three projections onto two factors is injective. By taking suitable quotients, it is possible to restrict our investigations to 2-factor injective subdirect products (see Lemma 3.7), for which we obtain the following theorem.

THEOREM 1.1 (Characterization of 2-factor injective subdirect products of 3 -factor products). Let $\Delta \leq G_{1} \times G_{2} \times G_{3}$ be a 2-factor injective subdirect product. Then there is a normal subgroup $H \unlhd \Delta$ with $\left[\pi_{i}(\Delta): \pi_{i}(H)\right]=[\Delta: H]$ for $i \in\{1,2,3\}$ and $H$ is isomorphic to a group of the following form: there are three finite groups $H_{1}, H_{2}, H_{3}$ that all have an Abelian subgroup $M$ contained in their center such that $H$ is isomorphic to the factor group of those triples $\left\{\left(\left(h_{2}, h_{3}^{\prime-1}\right),\left(h_{3}, h_{1}^{\prime-1}\right),\left(h_{1}, h_{2}^{\prime-1}\right)\right)\right\}$ that satisfy $h_{i}, h_{i}^{\prime} \in H_{i}, h_{i} h_{i}^{\prime-1} \in M$ and $h_{1} h_{1}^{\prime-1} h_{2} h_{2}^{\prime-1} h_{3} h_{3}^{\prime-1}=1$, by the normal subgroup

$$
\left\{\left(\left(m_{1}, m_{1}\right),\left(m_{2}, m_{2}\right),\left(m_{3}, m_{3}\right)\right) \mid m_{i} \in M\right\} .
$$

In this theorem $H$ is the subgroup

$$
\left\langle\left\{\left(g_{1}, g_{2}, g_{3}\right) \in \Delta \mid \exists i \in\{1,2,3\} \text { s.t. } g_{i}=1\right\}\right\rangle
$$

generated by all elements of $\Delta$ for which some entry is trivial. 


\section{SUBGROUPS OF 3-FACTOR DIRECT PRODUCTS}

Our intuitive interpretation of this theorem is as follows. The coset of $H$ in which an element of $\left(g_{1}, g_{2}, g_{3}\right) \in \Delta$ is contained is already determined by each of the three components $g_{i}$ alone. Moreover, the structure of $H$ is entirely determined by pairwise dependencies that are shared between two of the three factors $G_{1}, G_{2}, G_{3}$ but are independent of the third, together with an Abelian entanglement of all three factors, which is controlled by the subgroup $M$.

In the spirit of a well known correspondence version of Goursat's Lemma (see Theorem 2.2) we then investigate correspondence theorems between data obtained from the subgroup lattices of the $G_{i}$ (as well as isomorphisms between arising factor groups) and the subdirect products of $G_{1} \times G_{2} \times G_{3}$. For two special cases, namely the cases $H=\Delta$, and $M=\{1\}$, we obtain complete correspondence theorems for three factors (cf. Theorem 3.11 and Corollary 3.16). Here, the second case is a particular special case hinted at in [2]. In fact, the authors state in 2 that it is very likely that this case is describable by a symmetric version of a generalized Goursat's Lemma, and our Corollary 3.16 confirms this.

In a third special case, where one of the $G_{i}$ is the semidirect product of the projection of $H$ onto the $i$-th component and some other group, we also obtain a partial correspondence theorem (Theorem 3.17).

As demonstrated by P e t r i 11 o [13], Goursat's Lemma can readily be applied to count subgroups of the product of two Abelian groups. Some refined formulas were given by $\mathrm{T} \breve{\mathrm{r} n} \mathrm{n} \breve{u} \mathrm{c}$ e a n u [16] and Tót h [15]. For a direct product of an arbitrary number of Abelian groups the number of subgroups has been extensively studied. We refer to the monograph of B u t le r [5]. In fact there are also explicit formulas for the counts of subgroups of $\mathbb{Z}_{n} \times \mathbb{Z}_{m} \times \mathbb{Z}_{r}$ (see, for example [10). In line with the papers and as an application of our characterization, we derive an explicit formula to count the number of subdirect products of the direct product of three symmetric groups $S_{n_{1}} \times S_{n_{2}} \times S_{n_{3}}$.

THEOREM 1.2. Let $n_{1} \geq n_{2} \geq n_{3} \geq 2, n_{1} \geq 5$. For the number $\ell\left(n_{1}, n_{2}, n_{3}\right)$ of subdirect products of $S_{n_{1}} \times S_{n_{2}} \times S_{n_{3}}$ we have $\ell\left(n_{1}, n_{2}, n_{3}\right)=$

$$
\begin{cases}\left(n_{1} !\right)^{2}+6 n_{1} !+6 & \text { if } n_{1}=n_{2}=n_{3} \notin\{6\}, \\ 2082246 & \text { if } n_{1}=n_{2}=n_{3}=6, \\ 66 & \text { if } n_{2}=n_{3}=4, \\ 18 & \text { if } n_{2} \in\{3,4\}, n_{3}=3, \\ 2886 & \text { if }\left\{\left\{n_{1}, n_{2}, n_{3}\right\}=\left\{\left\{6,6, m_{2}\right\}, m_{2} \neq 6,\right.\right. \\ 2 m_{1} !+6 & \text { if }\left\{\left\{n_{1}, n_{2}, n_{3}\right\}\right\}=\left\{m_{1}, m_{1}, m_{2}\right\}, m_{1} \neq m_{2}, 6 \neq m_{1} \geq 5, \\ 6, & \text { otherwise. }\end{cases}
$$


The finitely many cases not covered by the Theorem are listed in Table 1 .

TABLE 1 . The numbers $\ell\left(n_{1}, n_{2}, n_{3}\right)$ for $n_{1}, n_{2}, n_{3} \in\{1,2,3,4\}$.

\begin{tabular}{c||c|c|c|c}
$n_{1}=4$ & 4 & 3 & 2 & 1 \\
\hline \hline 4 & 1386 & 282 & 66 & 32 \\
\hline 3 & 282 & 90 & 18 & 8 \\
\hline 2 & 66 & 18 & 6 & 2 \\
\hline 1 & 32 & 8 & 2 & 1
\end{tabular}

\begin{tabular}{c||c|c|c}
$n_{1}=3$ & 3 & 2 & 1 \\
\hline \hline 3 & 90 & 18 & 8 \\
\hline 2 & 18 & 6 & 2 \\
\hline 1 & 8 & 2 & 1
\end{tabular}

\begin{tabular}{c||c|c}
$n_{1}=2$ & 2 & 1 \\
\hline \hline 2 & 6 & 2 \\
\hline 1 & 2 & 1
\end{tabular}

It is also possible for example to count the normal subgroups of such direct products. In fact, the normal subgroups can be also characterized for arbitrary finite products of symmetric groups [12]. Let us finally also point to some literature concerning finiteness properties of groups [3, 4] which also contains some structural results on 3 -factor direct products (in particular on the case we call 2-factor surjective).

While the examples from [2] mentioned above stand in the way of a general correspondence theorem based on data coming from certain subgroup lattices of the factors and isomorphism between arising factor groups, the question remains whether a reasonable general correspondence theorem can be based on other suitable data.

\section{Goursat's Lemma}

Let $G=G_{1} \times G_{2} \times \cdots \times G_{t}$ be a direct product of groups and let $\Delta \leq G$ be a subgroup. We define for $i \in\{1, \ldots, t\}$ the map $\pi_{i}$ as the projection to the $i$-th coordinate and we define the homomorphism $\psi_{i}: \Delta \rightarrow G_{1} \times \cdots \times G_{i-1} \times G_{i+1} \times$ $\cdots \times G_{t}:\left(g_{1}, g_{2}, \ldots, g_{t}\right) \mapsto\left(g_{1}, \ldots, g_{i-1}, g_{i+1}, \ldots, g_{t}\right)$. If $G_{1}=G_{2}=\cdots=G_{t}$, then the subgroup consisting of the elements $\left\{(g, g, \ldots, g) \mid g \in G_{1}\right\}$ is called the diagonal subgroup.

A subgroup $\Delta \leq G$ of the direct product is said to be a subdirect product if $\pi_{i}(\Delta)=G_{i}$ for all $1 \leq i \leq t$. Goursat's Lemma is a classic statement concerned with the structure of subdirect products of direct products of two factors.

TheOREM 2.1 (Goursat's Lemma). Let $\Delta \leq G_{1} \times G_{2}=G$ be a subdirect product and define $N_{1}=\left\{g_{1} \in G_{1} \mid\left(g_{1}, 1\right) \in \Delta\right\}$ as well as $N_{2}=\left\{g_{2} \in G_{2} \mid\right.$ $\left.\left(1, g_{2}\right) \in \Delta\right\}$. Then $G_{1} / N_{1}$ is isomorphic to $G_{2} / N_{2}$ via an isomorphism $\varphi$ for which $\left(g_{1}, g_{2}\right) \in \Delta$ if and only if $\varphi\left(g_{1}\right)=g_{2}$. 


\section{SUBGROUPS OF 3-FACTOR DIRECT PRODUCTS}

This gives a natural homomorphism $\Delta \rightarrow G_{1} / N_{1} \times G_{2} / N_{2}$ that is defined as $\left(g_{1}, g_{2}\right) \mapsto\left(g_{1} N_{1}, g_{2} N_{2}\right)$ with image $\left\{\left(g_{1} N_{1}, g_{2} N_{2}\right) \mid \varphi\left(g_{1}\right)=g_{2}\right\}$. Thus we can view $\Delta$ as a fiber product (or pull back) of $G_{1}$ and $G_{2}$ over $G_{i} / N_{i}$.

A typical application of the lemma is a proof of the fact that subdirect products of non-Abelian finite simple groups are isomorphic to direct products of diagonal subgroups. Furthermore, the lemma can be applied to count (not necessarily subdirect) subgroups of direct products. For this, the following well known correspondence version of the lemma is more convenient.

THEOREM 2.2. There is a natural one-to-one correspondence between the subgroups of $G_{1} \times G_{2}$ and the tuples $\left(P_{1}, P_{2}, N_{1}, N_{2}, \varphi\right)$ for which for $i \in\{1,2\}$ we have:

(1) $N_{i} \unlhd P_{i} \leq G_{i}$ and

(2) $P_{1} / N_{1} \stackrel{\varphi}{\cong} P_{2} / N_{2}$.

Here, we write $G_{1} \stackrel{\varphi}{\cong} G_{2}$ to denote that $G_{1}$ and $G_{2}$ are isomorphic via an isomorphism $\varphi$. The subdirect products correspond to those tuples for which $P_{1}=G_{1}$ and $P_{2}=G_{2}$. Diagonal subgroups are those subdirect products that also satisfy $N_{1}=N_{2}=1$. Subproducts (i.e., direct products of a subgroup of $G_{1}$ with a subgroup of $G_{2}$ ) are those for which $N_{1}=P_{1}$ and $N_{2}=P_{2}$.

\section{Three factors}

We now focus on 3 -factor subdirect products. Before we investigate the general case, we consider four examples of subdirect products.

In our first example, we consider groups that are 2-factor surjective. We say $\Delta \leq G_{1} \times G_{2} \times G_{3}$ is 2-factor surjective if $\psi_{i}$ is surjective for all $1 \leq i \leq 3$. Note that the analogous definition of 1-factor surjectivity (i.e., all $\pi_{i}$ are surjective) means then the same as being subdirect.

Similarly, we say $\Delta$ is 2 -factor injective if $\psi_{i}$ is injective for all $1 \leq i \leq 3$. Note that this assumption is equivalent to saying that two components of an element of $\Delta$ determine the third. Analogously 1-factor injective then means that one component determines the other two.

\subsection{Examples of 3-factor direct products}

Example 3.1. The subgroup of $\left(G_{1}\right)^{3}$ comprised of the set $\left.\left\{(g, g, g) \mid g \in G_{1}\right)\right\}$ is called the diagonal subgroup.

It is not difficult to see that all 1-factor injective subdirect products are isomorphic to a diagonal subgroup. As a second example, let $G_{1}$ be an Abelian group. Then the group 


\section{DANIEL NEUEN-PASCAL SCHWEITZER}

EXAMPLE 3.2 .

$$
\Delta:=\left\{(a, b, c) \in\left(G_{1}\right)^{3} \mid a b c=1\right\}
$$

is a subdirect product of $\left(G_{1}\right)^{3}$ that is 2-factor surjective and 2-factor injective.

It turns out that this is essentially the only type of group with these properties, as argued by the following lemma.

LEMMA 3.3. Let $G=G_{1} \times G_{2} \times G_{3}$ be a group and $\Delta$ a subdirect product of $G$ that is 2-factor surjective and 2-factor injective. Then $G_{1}, G_{2}$ and $G_{3}$ are isomorphic Abelian groups and $\Delta$ is isomorphic to the subgroup of $G_{1}^{3}$ given by $\left\{(a, b, c) \in\left(G_{1}\right)^{3} \mid a b c=1\right\}$, which in turn is isomorphic to $\left(G_{1}\right)^{2}$ as an abstract group.

P r o of. Let $g_{1}$ and $g_{1}^{\prime}$ be elements of $G_{1}$. Then by 2-factor surjectivity there are elements $g_{2}$ and $g_{3}$ such that $\left(g_{1}, g_{2}, 1\right) \in \Delta$ and $\left(g_{1}^{\prime}, 1, g_{3}\right) \in \Delta$. We thus have that $\left(g_{1}, g_{2}, 1\right)^{\left(g_{1}^{\prime}, 1, g_{3}\right)}=\left(g_{1}^{g_{1}^{\prime}}, g_{2}, 1\right)$. By 2-factor injectivity it follows that $g_{1}^{g_{1}^{\prime}}=g_{1}$ and thus $G_{1}$ is Abelian.

For every $g_{1} \in G_{1}$ there is exactly one element of the form $\left(g_{1}, g_{2}, 1\right)$ in $\Delta$. The map $\varphi$ that sends every $g_{1}$ to the corresponding $g_{2}$ provides us with a map from $G_{1}$ to $G_{2}$. By 2-factor surjectivity and 2-factor injectivity, this map is an isomorphism from $G_{1}$ to $G_{2}$. Similarly, $G_{1}$ and $G_{3}$ are isomorphic.

Finally, note that the map sending $\left(g_{1}, g_{2}, g_{3}\right)$ to $\left(g_{1}, \varphi^{-1}\left(g_{2}\right)^{-1}, g_{1}^{-1} \varphi^{-1}\left(g_{2}\right)\right)$ is an isomorphism from $\Delta$ to $\left\{(a, b, c) \in\left(G_{1}\right)^{3} \mid a b c=1\right\}$.

We now drop the requirement for the group to be 2-factor surjective. Our next examples of 2-factor injective subdirect products will be non-Abelian.

EXAmple 3.4. Let $G_{1}=H \rtimes K$ be a semidirect product with an Abelian normal subgroup $H$. Then

$$
\Delta=\left\{(a k, b k, c k) \in\left(G_{1}\right)^{3} \mid a, b, c \in H, k \in K, a b c=1\right\}
$$

is a 2-factor injective subdirect product of $\left(G_{1}\right)^{3}$. To see this we verify that $\Delta$ is closed under multiplication. Let $d=(a k, b k, c k), d^{\prime}=\left(a^{\prime} k^{\prime}, b^{\prime} k^{\prime}, c^{\prime} k^{\prime}\right) \in \Delta$. Then

$$
\begin{aligned}
d d^{\prime} & =(a k, b k, c k)\left(a^{\prime} k^{\prime}, b^{\prime} k^{\prime}, c^{\prime} k^{\prime}\right) \\
& =\left(a k a^{\prime} k^{\prime}, b k b^{\prime} k^{\prime}, c k c^{\prime} k^{\prime}\right) \\
& =\left(a\left(k a^{\prime} k^{-1}\right) k k^{\prime}, b\left(k b^{\prime} k^{-1}\right) k k^{\prime}, c\left(k c^{\prime} k^{-1}\right) k k^{\prime}\right)
\end{aligned}
$$

and $a\left(k a^{\prime} k^{-1}\right) b\left(k b^{\prime} k^{-1}\right) c\left(k c^{\prime} k^{-1}\right)=(a b c) k\left(a^{\prime} b^{\prime} c^{\prime}\right) k^{-1}=1$ implying $d d^{\prime} \in \Delta$. So $\Delta \leq\left(G_{1}\right)^{3}$. The fact that $\Delta$ is a subdirect product and 2-factor injective follows directly from the definition. 
ExAmple 3.5. As a next example suppose $G_{1}=H_{2} \times H_{3}, G_{2}=H_{1} \times H_{3}$ and $G_{3}=H_{1} \times H_{2}$ with arbitrary finite groups $H_{i}$. Then the group consisting of the set

$$
\left\{\left(\left(h_{2}, h_{3}\right),\left(h_{1}, h_{3}\right),\left(h_{1}, h_{2}\right)\right) \mid h_{i} \in H_{i}\right\}
$$

is a 2-factor injective subdirect product of $G_{1} \times G_{2} \times G_{3}$.

Finally, it is not difficult to construct subdirect products that are not 2-factor injective by considering extensions of the factors.

EXAMPLE 3.6 . Let $\Delta \leq G_{1} \times G_{2} \times G_{3}$ be a subdirect product and $\kappa: \widetilde{G_{1}} \rightarrow G_{1}$ a surjective homomorphism. Then $\left\{\left(g_{1}, g_{2}, g_{3}\right) \in \widetilde{G_{1}} \times G_{2} \times G_{3} \mid\left(\kappa\left(g_{1}\right), g_{2}, g_{3}\right) \in \Delta\right\}$ is a subdirect product of $\widetilde{G_{1}} \times G_{2} \times G_{3}$ that is not 2-factor injective if $\kappa$ is not injective.

\subsection{The structure of subgroups of 3 -factor direct products}

We now analyze the general case, showing that it must essentially be a combination of the examples presented above. We first argue that we can focus our attention on 2-factor injective subdirect products.

LemMA 3.7. Let $\Delta \leq G_{1} \times G_{2} \times G_{3}$ be a subdirect product. Further define $N_{i}:=$ $\pi_{i}\left(\operatorname{ker}\left(\psi_{i}\right)\right)$ for $i \in\{1,2,3\}$. Then $\Delta^{\prime}=\Delta /\left(N_{1} \times N_{2} \times N_{3}\right)$ is a 2-factor injective subdirect product and $\Delta=\left\{\left(g_{1}, g_{2}, g_{3}\right) \mid\left(g_{1} N_{1}, g_{2} N_{2}, g_{3} N_{3}\right) \in \Delta^{\prime}\right\}$.

Thus in the following suppose $\Delta$ is a 2-factor injective subdirect product of $G_{1} \times G_{2} \times G_{3}$.

Let $H_{i}=\operatorname{ker}\left(\pi_{i}\right) \cap \Delta=\left\{\left(g_{1}, g_{2}, g_{3}\right) \in \Delta \mid g_{i}=1\right\}$. Then $H=\left\langle H_{1}, H_{2}, H_{3}\right\rangle$ is a normal subgroup of $\Delta$.

Lemma 3.8. For $i, j \in\{1,2,3\}$ with $i \neq j$ we have $\left[H_{i}, H_{j}\right]=1$, that is, all elements in $H_{i}$ commute with all elements in $H_{j}$.

P r o of. Without loss of generality assume that $i=1$ and $j=2$. For $\left(1, g_{2}, g_{3}\right) \in$ $H_{1}$ and $\left(h_{1}, 1, h_{3}\right)$ in $H_{2}$ we get that $\left(1, g_{2}, g_{3}\right)^{\left(h_{1}, 1, h_{3}\right)}=\left(1, g_{2}, g_{3}^{h_{3}}\right)$. By 2 -factor injectivity we conclude that $g_{3}^{h_{3}}=g_{3}$ and thus the two elements commute.

Define $M_{i}:=\pi_{i}\left(H_{k}\right) \cap \pi_{i}\left(H_{j}\right)$, where $j$ and $k$ are chosen so that $\{i, j, k\}=$ $\{1,2,3\}$.

Lemma 3.9. Let $i, j, k$ be integers such that $\{i, j, k\}=\{1,2,3\}$. Then there is a canonical isomorphism $\varphi:=\varphi_{j, k}^{i}$ from $\pi_{j}\left(H_{i}\right)$ to $\pi_{k}\left(H_{i}\right)$ that maps $M_{j}$ to $M_{k}$.

P r o of. Assume without loss of generality that $i=1, j=2$ and $k=3$. Define a map $\varphi: \pi_{2}\left(H_{1}\right) \rightarrow \pi_{3}\left(H_{1}\right)$ such that $\left(1, g_{2}, \varphi\left(g_{2}\right)^{-1}\right) \in \Delta$ for all $g_{2} \in \pi_{2}\left(H_{1}\right)$. 
Such a map exists and is well defined since $\Delta$ is a 2-factor injective subdirect product. Suppose $g_{2} \in M_{2}$ then $\left(1, g_{2}, \varphi\left(g_{2}\right)^{-1}\right) \in \Delta$ and there is a $g_{1}$ such that $\left(g_{1}, g_{2}, 1\right) \in \Delta$. Then $\left(1, g_{2}, \varphi\left(g_{2}\right)^{-1}\right)\left(g_{1}, g_{2}, 1\right)^{-1}=\left(g_{1}^{-1}, 1, \varphi\left(g_{2}\right)^{-1}\right)$ so $\varphi\left(g_{2}\right) \in M_{3}$. It follows by symmetry that all $M_{i}$ are isomorphic and that $\left.\varphi\right|_{M_{2}}$ is an isomorphism from $M_{2}$ to $M_{3}$.

Note that the canonical isomorphisms behave well with respect to composition. In particular we have $\varphi_{j, k}^{i}=\left(\varphi_{k, j}^{i}\right)^{-1}$ and $\left.\left.\varphi_{j, k}^{i}\right|_{M_{j}} \circ \varphi_{k, i}^{j}\right|_{M_{k}}=\left.\varphi_{j, i}^{k}\right|_{M_{j}}$. This implies for example that the composition of the canonical isomorphism from $M_{1}$ to $M_{2}$ and the canonical isomorphism from $M_{2}$ to $M_{3}$ is exactly the canonical isomorphism from $M_{1}$ to $M_{3}$. We can thus canonically identify the subgroups $M_{1}, M_{2}$ and $M_{3}$ with a fixed subgroup $M$.

Moreover, we can canonically associate the elements in $H_{1}$ with the elements in $\pi_{2}\left(H_{1}\right)$ and with the elements in $\pi_{3}\left(H_{1}\right)$ by associating $\left(1, g_{2}, \varphi\left(g_{2}\right)^{-1}\right) \in H_{1}$ with the element $g_{2} \in G_{2}$ and $\varphi\left(g_{2}\right)$ in $G_{3}$. Similarly we can associate elements $\left(\varphi\left(g_{3}\right)^{-1}, 1, g_{3}\right) \in H_{2}$ with $g_{3} \in G_{3}$ and $\varphi\left(g_{3}\right) \in G_{1}$ and also associate $\left(g_{1}, \varphi\left(g_{1}\right)^{-1}, 1\right) \in H_{3}$ with $g_{1} \in G_{1}$ and $\varphi\left(g_{1}\right) \in G_{2}$.

THEOREM 1.1 (RESTATED). Let $\Delta \leq G_{1} \times G_{2} \times G_{3}$ be a 2-factor injective subdirect product. Then there is a normal subgroup $H \unlhd \Delta$ with $\left[\pi_{i}(\Delta): \pi_{i}(H)\right]=$ $[\Delta: H]$ for $i \in\{1,2,3\}$ and $H$ is isomorphic to a group of the following form: there are three finite groups $H_{1}, H_{2}, H_{3}$ that all have an Abelian subgroup $M$ contained in their center such that $H$ is isomorphic to the factor group of triples $\left\{\left(\left(h_{2}, h_{3}^{\prime-1}\right),\left(h_{3}, h_{1}^{\prime-1}\right),\left(h_{1}, h_{2}^{\prime-1}\right)\right)\right\}$ that satisfy $h_{i}, h_{i}^{\prime} \in H_{i}, h_{i} h_{i}^{\prime-1} \in M$ and $h_{1} h_{1}^{\prime-1} h_{2} h_{2}^{\prime-1} h_{3} h_{3}^{\prime-1}=1$, by the normal subgroup

$$
\left\{\left(\left(m_{1}, m_{1}\right),\left(m_{2}, m_{2}\right),\left(m_{3}, m_{3}\right)\right) \mid m_{i} \in M\right\} .
$$

Proof. As before define $H_{i}=\operatorname{ker}\left(\pi_{i}\right)=\left\{\left(g_{1}, g_{2}, g_{3}\right) \in \Delta \mid g_{i}=1\right\}$ and $H=$ $\left\langle H_{1}, H_{2}, H_{3}\right\rangle$. By Lemma 3.9 and the comment about the compatibility of the isomorphisms between the groups we can canonically associate the elements of $M_{i}$ with those of $M_{j}$. Moreover we can assume that there is an Abelian group $M$ that is isomorphic to the intersection of every pair of $\left\{H_{1}, H_{2}, H_{3}\right\}$. All elements of $H$ commute with all elements in such an intersection.

If $\left(g_{1}, g_{2}, g_{3}\right)$ is an element of $H$, then each $g_{i}$ can be written as $c_{i} \cdot b_{i}^{-1}$ with $c_{i} \in$ $C_{i}:=\pi_{i}\left(H_{i+1}\right)$ and $b_{i} \in B_{i}:=\pi_{i}\left(H_{i+2}\right)$, where indices will always be taken modulo 3. We can set $\left(g_{1}, g_{2}, g_{3}\right)=\left(c_{1} b_{1}^{-1}, c_{2} b_{2}^{-1}, c_{3} b_{3}^{-1}\right)$. Since $c_{1} \in \pi_{1}\left(H_{2}\right)$ we conclude that $\left(c_{1}^{-1}, 1, \varphi_{1,3}^{2}\left(c_{1}\right)\right) \in H$. We also have $\left(b_{1}, \varphi_{1,2}^{3}\left(b_{1}\right)^{-1}, 1\right) \in H$. This implies that the product $\left(g_{1}, g_{2}, g_{3}\right)\left(c_{1}^{-1}, 1, \varphi_{1,3}^{2}\left(c_{1}\right)\right)\left(b_{1}, \varphi_{1,2}^{3}\left(b_{1}{ }^{-1}\right), 1\right)$ is equal to $\left(1, c_{2} b_{2}^{-1} \varphi_{1,2}^{3}\left(b_{1}^{-1}\right), c_{3} b_{3}^{-1} \varphi_{1,3}^{2}\left(c_{1}\right)\right) \in H$. 


\section{SUBGROUPS OF 3-FACTOR DIRECT PRODUCTS}

We thus see by looking at the third component that $c_{3} b_{3}{ }^{-1} \varphi_{1,3}^{2}\left(c_{1}\right) \in \pi_{3}\left(H_{1}\right)$ which implies that $b_{3}{ }^{-1} \varphi_{1,3}^{2}\left(c_{1}\right) \in \pi_{3}\left(H_{1}\right)$ and thus $b_{3}{ }^{-1} \varphi_{1,3}^{2}\left(c_{1}\right) \in M_{3}$. By symmetry we conclude that

$$
b_{i+1}^{-1} \varphi_{i-1, i+1}^{i}\left(c_{i-1}\right) \in M_{i+1} \text { for } i \in\{1,2,3\} .
$$

Looking at the second component, we also see that $c_{2} b_{2}{ }^{-1} \varphi_{1,2}^{3}\left(b_{1}{ }^{-1}\right) \in \pi_{2}\left(H_{1}\right)$ and in turn we conclude that

$$
\varphi_{2,3}^{1}\left(c_{2} b_{2}{ }^{-1} \varphi_{1,2}^{3}\left(b_{1}{ }^{-1}\right)\right)^{-1}=c_{3} b_{3}{ }^{-1} \varphi_{1,3}^{2}\left(c_{1}\right) .
$$

Recalling that $H_{i}$ and $H_{j}$ commute for $i \neq j$, we thus conclude that

$$
c_{3} \varphi_{2,3}^{1}\left(b_{2}\right)^{-1} \cdot \varphi_{1,3}^{2}\left(c_{1}\right) b_{3}{ }^{-1} \cdot \varphi_{2,3}^{1}\left(c_{2} \varphi_{1,2}^{3}\left(b_{1}{ }^{-1}\right)\right)=1 .
$$

Thus, since all involved isomorphisms are compatible we can reinterpret this equation in $M$ and we see that $c_{3}\left(b_{2}\right)^{-1} \cdot c_{1}\left(b_{3}\right)^{-1} \cdot c_{2}\left(b_{1}\right)^{-1}=1$.

Suppose that $\left(g_{1}, g_{2}, g_{3}\right)=\left({\widehat{c_{1}}}_{b_{1}}^{-1},{\widehat{c_{2}}}_{\hat{b}_{2}}^{-1},{\widehat{c_{3}}}^{-1}{\widehat{b_{3}}}^{-1}\right)$ is a second representation of the element $\left(g_{1}, g_{2}, g_{3}\right)$ of $H$. Then we have for the first component that $\widehat{c_{1}}{\widehat{b_{1}}}^{-1}=c_{1} b_{1}{ }^{-1}$ and thus $c_{1}^{-1} \widehat{c_{1}}=b_{1}{ }^{-1} \widehat{b_{1}} \in M_{1}$ or, in other words, $\widehat{c_{1}} m_{1}=$ $c_{1}$ and ${\widehat{b_{1}}}^{-1} m_{1}=b_{1}^{-1}$ for some element $m_{1} \in M$. Similarly there are elements $m_{2}$ and $m_{3}$ for the other components. We conclude that the map sending $\left(g_{1}, g_{2}, g_{3}\right)$ to $\left(\left(c_{1}, b_{1}{ }^{-1}\right),\left(c_{2}, b_{2}{ }^{-1}\right),\left(c_{3}, b_{3}{ }^{-1}\right)\right)$ is a homomorphism from $H$ to the group described in the theorem.

It remains to show injectivity of this homomorphism. Thus, suppose that the triple $\left(g_{1}, g_{2}, g_{3}\right)$ is mapped to the trivial element. This implies for the first component of the image $\left(\left(c_{1}, b_{1}^{-1}\right),\left(c_{2}, b_{2}{ }^{-1}\right),\left(c_{3}, b_{3}{ }^{-1}\right)\right)$ that $\left(c_{1}, b_{1}^{-1}\right)=\left(m_{1}, m_{1}\right)$ for some $m_{1}$ implying that $g_{1}=m_{1} m_{1}{ }^{-1}=1$. Repeating the same argument for the other components we see that the homomorphism is an isomorphism.

\subsection{Correspondence theorems}

We now investigate the possibility of having a correspondence theorem in the style of Theorem 2.2 for 3 factors. As before, we can readily reduce to the case of 2-factor injective subdirect products.

LEMMA 3.10. There is a natural one-to-one correspondence between subdirect products of $G_{1} \times G_{2} \times G_{3}$ and the tuples $\left(N_{1}, N_{2}, N_{3}, \Delta^{\prime}\right)$, where $N_{i} \unlhd G_{i}$ for every $i \in\{1,2,3\}$ and $\Delta^{\prime}$ is a 2-factor injective subdirect product of $G_{1} / N_{1} \times G_{2} / N_{2} \times$ $G_{3} / N_{3}$.

P r o o f. Let $\Delta \leq G_{1} \times G_{2} \times G_{3}$ be a subdirect product. Choose $N_{i}=\pi_{i}\left(\operatorname{ker}\left(\psi_{i}\right)\right)$ for $i \in\{1,2,3\}$ and let $\Delta^{\prime}=\Delta /\left(N_{1} \times N_{2} \times N_{3}\right)$. Then $N_{i} \unlhd G_{i}$, because $\Delta$ is a subdirect product, and $\Delta^{\prime}$ is 2-factor injective by Lemma 3.7 


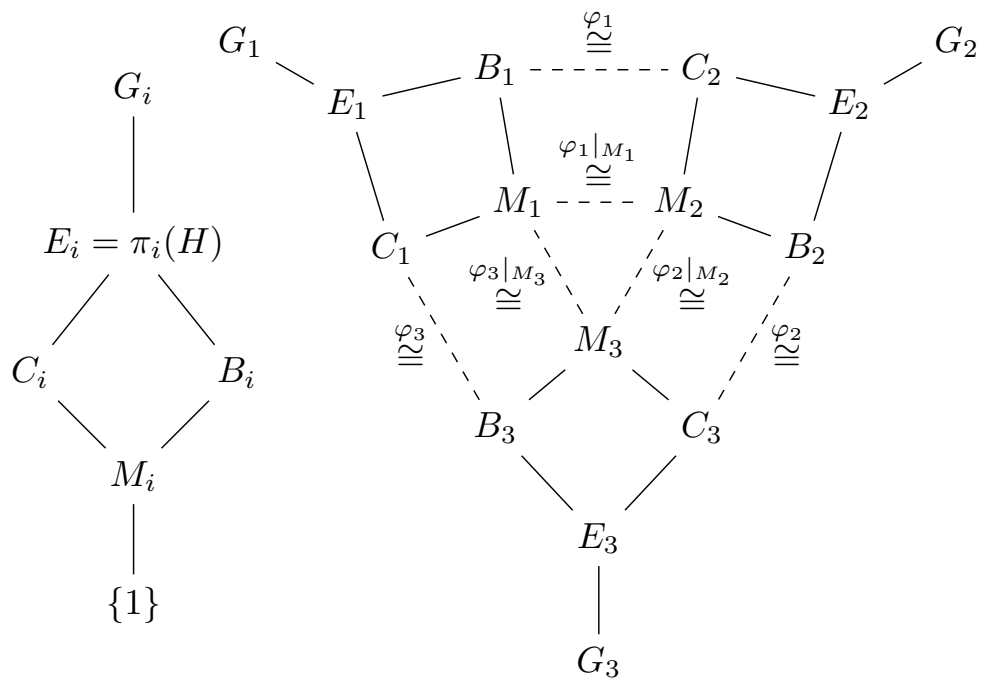

Figure 3.1. Subgroup diagram for the group $G_{i}$ (left), and connections between the subgroups of the projections to the different components (right).

Conversely, let $N_{i} \unlhd G_{i}$ and let $\Delta^{\prime}$ be a 2-factor injective subdirect product of $G_{1} / N_{1} \times G_{2} / N_{2} \times G_{3} / N_{3}$. Then $\Delta=\left\{\left(g_{1}, g_{2}, g_{3}\right) \mid\left(g_{1} N_{1}, g_{2} N_{2}, g_{3} N_{3}\right) \in \Delta^{\prime}\right\}$ is subdirect product of $G_{1} \times G_{2} \times G_{3}$.

Suppose $\Delta$ is a 2-factor injective subdirect product. Then we can define for $i \in$ $\{1,2,3\}$ the groups $H_{i}=\left\{\left(g_{1}, g_{2}, g_{3}\right) \in \Delta \mid g_{i}=1\right\}$ and with them the groups $B_{i}=\pi_{i}\left(H_{i+2}\right)$ and $C_{i}=\pi_{i}\left(H_{i+1}\right)$. As we will see, the canonical isomorphism $\varphi^{i}:=\varphi_{i, i+1}^{i+2}$ that exists by Lemma 3.9 can be extended to an isomorphism from $G_{i} / C_{i}$ to $G_{i+1} / B_{i+1}$. We would like to have a correspondence theorem in the style of Theorem 2.2 for 3 factors. For this, in principle, we would like to relate the 2 -factor injective subdirect products of $G_{1}, G_{2}, G_{3}$ to the tuples

$$
\left(B_{1}, B_{2}, B_{3}, C_{1}, C_{2}, C_{3}, \varphi_{1}, \varphi_{2}, \varphi_{3}\right)
$$

that satisfy certain consistency properties. However, in general, neither is it clear which consistency properties to choose so that every tuple corresponds to a subdirect product, nor do distinct subdirect products always correspond to distinct tuples. Indeed, in [2] the authors describe two distinct Abelian subdirect products of the same group $G_{1} \times G_{2} \times G_{3}$ for which the corresponding tuples agree.

In the light of that we content ourselves with studying two special cases, namely those where $\Delta=H$ and those where $M_{i}=B_{i} \cap C_{i}=1$. 


\section{SUBGROUPS OF 3-FACTOR DIRECT PRODUCTS}

THEOREM 3.11. There is a natural one-to-one correspondence between subdirect products of $\Delta \leq G_{1} \times G_{2} \times G_{3}$ which are 2-factor injective satisfying $\Delta=H$, and tuples $\left(B_{1}, B_{2}, B_{3}, C_{1}, C_{2}, C_{3}, \varphi_{1}, \varphi_{2}, \varphi_{3}\right)$ for which for all $i \in\{1,2,3\}$ (indices taken modulo 3) we have

(1) $B_{i}, C_{i} \unlhd G_{i}$,

(2) $B_{i} C_{i}=G_{i}$,

(3) $B_{i} \stackrel{\varphi_{i}}{\cong} C_{i+1}$,

(4) $\left[B_{i}, C_{i}\right]=1$,

(5) $\varphi_{i}\left(B_{i} \cap C_{i}\right)=B_{i+1} \cap C_{i+1}$,

(6) $\left.\left.\left.\varphi_{3}\right|_{B_{3} \cap C_{3}} \circ \varphi_{2}\right|_{B_{2} \cap C_{2}} \circ \varphi_{1}\right|_{B_{1} \cap C_{1}}=i d$.

P r o o f. Let $\Delta \leq G_{1} \times G_{2} \times G_{3}$ be a subdirect product. Define $H_{i}:=\left\{\left(g_{1}, g_{2}, g_{3}\right) \in\right.$ $\left.\Delta \mid g_{i}=1\right\}$ for $i \in\{1,2,3\}$ and $H=\left\langle H_{1}, H_{2}, H_{3}\right\rangle$. Suppose that $H=\Delta$. For $i \in\{1,2,3\}$, define $B_{i}=\pi_{i}\left(H_{i+2}\right)$ and $C_{i}=\pi_{i}\left(H_{i+1}\right)$. Clearly $B_{i}, C_{i} \unlhd G_{i}$. By Lemma 3.8 we get that $\left[B_{i}, C_{i}\right]=1$ and $B_{i} C_{i}=\pi_{i}(H)$. The assumption $\Delta=H$ implies $B_{i} C_{i}=G_{i}$. By Lemma 3.9 the groups $B_{i}$ and $C_{i+1}$ are isomorphic via an isomorphism $\varphi_{i}=\varphi_{i, i+1}^{i+2}$, which maps $M_{i}=B_{i} \cap C_{i}$ to $M_{i+1}=B_{i+1} \cap C_{i+1}$. Finally, Property 3.11 follows directly from the comment below Lemma 3.9. This gives us the tuple $\left(B_{1}, B_{2}, B_{3}, C_{1}, C_{2}, C_{3}, \varphi_{1}, \varphi_{2}, \varphi_{3}\right)$ with the desired properties.

On the other hand, suppose we are given $\left(B_{1}, B_{2}, B_{3}, C_{1}, C_{2}, C_{3}, \varphi_{1}, \varphi_{2}, \varphi_{3}\right)$ with the desired properties. Let $M_{i}=B_{i} \cap C_{i}$. Define $\Delta$ to be the set of triples $\left(g_{1}, g_{2}, g_{3}\right) \in G_{1} \times G_{2} \times G_{3}$ that satisfy $g_{i}=c_{i} b_{i}^{-1}$ for $b_{i} \in B_{i}, c_{i} \in C_{i}, c_{i+1} M_{i+1}=$ $\varphi_{i}\left(b_{i} M_{i}\right)$ and $c_{3} \varphi_{2}\left(b_{2}\right)^{-1} \cdot \varphi_{3}^{-1}\left(c_{1}\right) b_{3}^{-1} \cdot \varphi_{2}\left(c_{2} \varphi_{1}\left(b_{1}{ }^{-1}\right)\right)=1$.

For $i \in\{1,2,3\}$ suppose $g_{i}=c_{i} b_{i}^{-1}=c_{i}^{\prime} b_{i}^{\prime-1}$. Then there is some $m_{i} \in M_{i}$ with $b_{i}=b_{i}^{\prime} m_{i}$ and $c_{i}=c_{i}^{\prime} m_{i}$. Hence, $b_{i} M_{i}=b_{i}^{\prime} M_{i}$ and $c_{i} M_{i}=c_{i}^{\prime} M_{i}$. Furthermore, we have

$$
\begin{aligned}
& c_{3}^{\prime} \varphi_{2}\left(b_{2}^{\prime}\right)^{-1} \cdot \varphi_{3}^{-1}\left(c_{1}^{\prime}\right) b_{3}^{\prime-1} \cdot \varphi_{2}\left(c_{2}^{\prime} \varphi_{1}\left(b_{1}^{\prime}{ }^{-1}\right)\right) \\
& =c_{3} m_{3}^{-1} \varphi_{2}\left(b_{2} m_{2}^{-1}\right)^{-1} \cdot \varphi_{3}^{-1}\left(c_{1} m_{1}^{-1}\right)\left(b_{3} m_{3}^{-1}\right)^{-1} \cdot \varphi_{2}\left(c_{2} m_{2}^{-1} \varphi_{1}\left(\left(b_{1} m_{1}^{-1}\right)^{-1}\right)\right) \\
& =c_{3} \varphi_{2}\left(b_{2}\right)^{-1} \cdot \varphi_{3}^{-1}\left(c_{1}\right) b_{3}^{-1} \cdot \varphi_{2}\left(c_{2} \varphi_{1}\left(b_{1}^{-1}\right)\right),
\end{aligned}
$$

so the membership in $\Delta$ is independent from the representation of $g_{i} \in G_{i}$. Also, it is easy to check that $\Delta$ is closed under multiplication because $\left[B_{i}, C_{i}\right]=1$. The group $\Delta$ is a subdirect product, since for $g_{1}=c_{1} b_{1}^{-1}$ we have that

$$
\left(c_{1} b_{1}^{-1}, \varphi_{1}\left(b_{1}^{-1}\right)^{-1} b_{2}^{-1}, \varphi_{3}^{-1}\left(c_{1}\right) \varphi_{2}\left(b_{2}\right)\right) \in \Delta,
$$




\section{DANIEL NEUEN-PASCAL SCHWEITZER}

and it can be checked that the group is 2-factor injective. Define

$$
H_{i}=\left\{\left(g_{1}, g_{2}, g_{3}\right) \in \Delta \mid g_{i}=1\right\} \quad \text { for } \quad i \in\{1,2,3\} \quad \text { and } \quad H=\left\langle H_{1}, H_{2}, H_{3}\right\rangle .
$$

Then $B_{i}=\pi_{i}\left(H_{i+2}\right)$ and $C_{i}=\pi_{i}\left(H_{i+1}\right)$, which means that $H=\Delta$ by Property 3.11 and Theorem [1.1. Finally, it can be checked that $\varphi_{i}=\varphi_{i, i+1}^{i+2}$.

It remains to show that for each 2-factor injective subdirect product $\Delta \leq$ $G_{1} \times G_{2} \times G_{3}$ with $\Delta=H$, the group $\Delta$ is of the form described above. But this follows from Theorem 1.1, Equation (3.3) and (3.4).

The previous theorem shows that for the subdirect products with $\Delta=H$ we can devise a correspondence theorem. As a second case, on the other end of he spectrum, we can also devise a correspondence theorem if the Abelian part that interlinks the three components is trivial. In fact this case corresponds to the case discussed by B a u e r, Sen, and Zvengrowski [2, 5.1 Remark], who already suspect that a theorem like the previous one can be obtained. We remark that Example 3.5 from the previous section is of this form. In fact, we can already conclude from Theorem 3.11 that for every group $\Delta$, where the Abelian part interlinking the components is trivial, the group $H$ essentially has the form of Example 3.5.

DEFINITION 3.12. Let $\Delta$ be a subdirect product of $G_{1} \times G_{2} \times G_{3}$. We say $\Delta$ is degenerate if $\pi_{i}\left(\operatorname{ker}\left(\pi_{i+1}\right)\right) \cap \pi_{i}\left(\operatorname{ker}\left(\pi_{i+2}\right)\right)=\pi_{i}\left(\operatorname{ker}\left(\psi_{i}\right)\right)$ (i.e., $\left.M_{i}=1\right)$ for some, and thus every, $i \in\{1,2,3\}$.

LEMMA 3.13. For $i \in\{1,2,3\}$ let $B_{i}, C_{i} \unlhd G_{i}$, such that

$$
B_{i} \cap C_{i}=1 \quad \text { and } \quad\left[B_{i}, C_{i}\right]=1 .
$$

Furthermore assume $G_{i} / C_{i} \stackrel{\varphi_{i}}{\cong} G_{i+1} / B_{i+1}$ and suppose

$$
\varphi_{i}\left(B_{i} C_{i}\right)=C_{i+1} B_{i+1} \text { and } \varphi_{3}\left(\varphi_{2} l\left(\varphi_{1}\left(g_{1} B_{1} C_{1}\right) l\right)\right)=g_{1} B_{1} C_{1} \text { for all } g_{1} \in G_{1} \text {. }
$$

Define

$$
\Delta=\left\{\left(g_{1}, g_{2}, g_{3}\right) \in G_{1} \times G_{2} \times G_{3} \mid \varphi_{i}\left(g_{i} C_{i}\right)=g_{i+1} B_{i+1}\right\} .
$$

Then $\pi_{i}(\Delta)=G_{i}$ and $\Delta$ is a degenerate 2-factor injective subdirect product.

P r o of. We first show, that $\Delta$ is closed under multiplication. For this let us assume that $\left(g_{1}, g_{2}, g_{3}\right),\left(g_{1}^{\prime}, g_{2}^{\prime}, g_{3}^{\prime}\right) \in \Delta$. Then $\varphi_{i}\left(g_{i} g_{i}^{\prime} C_{i}\right)=\varphi_{i}\left(g_{i} C_{i}\right) \varphi_{i}\left(g_{i}^{\prime} C_{i}\right)=$ $g_{i+1} g_{i+1}^{\prime} B_{i+1}$ for all $i \in\{1,2,3\}$, so $\left(g_{1} g_{1}^{\prime}, g_{2} g_{2}^{\prime}, g_{3} g_{3}^{\prime}\right) \in \Delta$. Let $E_{1}=\left\langle B_{1}, C_{1}\right\rangle$ and pick $e_{1} \in E_{1}$. The element $e_{1}$ can uniquely be written as $e_{1}=b_{1} c_{1}$ with $b_{1} \in B_{1}, c_{1} \in C_{1}$. For each $i \in\{1,2,3\}$ define $\varphi_{i}^{*}: B_{i} \rightarrow C_{i+1}$ with $\varphi_{i}^{*}\left(b_{i}\right)=c_{i+1}$ for the unique $c_{i+1} \in C_{i+1}$ with $\varphi_{i}\left(b_{i} C_{i}\right)=c_{i+1} B_{i+1}$. Then $\left(b_{1} c_{1}, b_{2} \varphi_{1}^{*}\left(b_{1}\right),\left(\varphi_{3}^{*}\right)^{-1}\left(c_{1}\right) \varphi_{2}^{*}\left(b_{2}\right)\right) \in \Delta$. So $E_{1} \leq \pi_{1}(\Delta)$. The argument for the other components is analogous. Now let $n_{1}^{1}, \ldots, n_{t}^{1}$ be a transversal of $E_{1}$ in $G_{1}$. Let $n_{i}^{2} B_{2}=\varphi_{1}\left(n_{i}^{1} C_{1}\right)$ and $n_{i}^{3} C_{3}=\varphi_{3}^{-1}\left(n_{i}^{1} B_{1}\right)$ for $i \in\{1, \ldots, t\}$. Then $\varphi_{2}\left(n_{i}^{2} C_{2}\right) \subseteq n_{i}^{3} E_{3}$ and hence there is some $b_{i}^{2} \in B_{2}$ with $\varphi_{2}\left(n_{i}^{2} b_{i}^{2} C_{2}\right)=n_{1}^{3} B_{3}$. 


\section{SUBGROUPS OF 3-FACTOR DIRECT PRODUCTS}

So $\left(n_{i}^{1}, n_{i}^{2} b_{i}^{2}, n_{i}^{3}\right) \in \Delta$ and $G_{1} \leq \pi_{1}(\Delta)$.

It remains to prove that $\Delta$ is 2-factor injective. Let $\left(g_{1}, g_{2}, g_{3}\right) \in \Delta$ with $g_{2}=g_{3}=1$. Then $g_{1} \in B_{1}$, because $\varphi_{3}\left(C_{3}\right)=B_{1}=g_{1} B_{1}$, and $g_{1} \in C_{1}$, because $\varphi_{1}\left(g_{1} C_{1}\right)=g_{2} B_{2}=B_{2}$. So $g_{1}=1$. Again, the argument for the other components is analogous.

LEMMA 3.14. Let $\Delta$ be a 2-factor injective subdirect product of $G_{1} \times G_{2} \times G_{3}$. Furthermore, let $H_{i}=\left\{\left(g_{1}, g_{2}, g_{3}\right) \in \Delta \mid g_{i}=1\right\}$ for $i \in\{1,2,3\}$ and $H=$ $\left\langle H_{1}, H_{2}, H_{3}\right\rangle$. Define

$$
B_{i}=\pi_{i}\left(H_{i+2}\right) \quad \text { and } \quad C_{i}=\pi_{i}\left(H_{i+1}\right) .
$$

Suppose

$$
B_{i} \cap C_{i}=1 \quad \text { for all } i \in\{1,2,3\} \text {. }
$$

Then there are canonical isomorphisms $\varphi_{1}, \varphi_{2}, \varphi_{3}$ such that $G_{i} / C_{i} \stackrel{\varphi_{i}}{\cong} G_{i+1} / B_{i+1}$ and $\varphi_{i}\left(B_{i} C_{i}\right)=C_{i+1} B_{i+1}$ such that $\varphi_{3}\left(\varphi_{2}\left(\varphi_{1}\left(g_{1} B_{1} C_{1}\right)\right)\right)=g_{1} B_{1} C_{1}$ for all $g_{1} \in G_{1}$. Furthermore $\Delta$ is given by Equation (3.5).

Proof. For $i \in\{1,2,3\}$ define a homomorphism $\varphi_{i}: G_{i} / C_{i} \rightarrow G_{i+1} / B_{i+1}$ by setting $\varphi_{i}\left(g_{i} C_{i}\right)=g_{i+1} B_{i+1}$ if $\left(g_{1}, g_{2}, g_{3}\right) \in \Delta$ for some $g_{i} \in G_{i}$. We first have to show that $\varphi_{i}$ is well-defined. Without loss of generality consider $i=1$ and let $\left(g_{1}, g_{2}, g_{3}\right),\left(g_{1}^{\prime}, g_{2}^{\prime}, g_{3}^{\prime}\right) \in \Delta$ with $g_{1} C_{1}=g_{1}^{\prime} C_{1}$. Then there is a $\left(c, 1, h_{2}\right) \in \Delta$ with $g_{1}^{\prime} c=g_{1}$. We obtain $\left(g_{1}^{\prime}, g_{2}^{\prime}, g_{3}^{\prime}\right)\left(c, 1, h_{2}\right)\left(g_{1}, g_{2}, g_{3}\right)^{-1}=$ $\left(1, g_{2}^{\prime} g_{2}^{-1}, g_{3}^{\prime \prime}\right)$ for some $g_{3}^{\prime \prime} \in G_{3}$ and hence, $g_{2} B_{2}=g_{2}^{\prime} B_{2}$. So $\varphi_{i}$ is well-defined. Since $\Delta$ is a subdirect product, $\varphi_{i}$ is a surjective homomorphism. Suppose $\varphi_{1}\left(g_{1} C_{1}\right)=B_{2}$. Then $\left(g_{1} c_{1}, b_{2}, g_{3}\right) \in \Delta$ for some $c_{1} \in C_{1}, b_{2} \in B_{2}$ and $g_{3} \in G_{3}$. Also there is $h_{3} \in G_{3}$ with $\left(1, b_{2}, h_{3}\right) \in \Delta$ and hence, $\left(g_{1} c_{1}, 1, g_{3} h_{3}^{-1}\right) \in \Delta$ implying that $g_{1} \in C_{1}$. So

$$
G_{i} / C_{i} \stackrel{\varphi_{i}}{\cong} G_{i+1} / B_{i+1}
$$

For every $b_{1} \in B_{1}$ there is a $c_{2} \in C_{2}$ with $\left(b_{1}, c_{2}, 1\right) \in \Delta$ and $\varphi_{1}\left(b_{1} C_{1}\right)=$ $c_{2} B_{2} \in C_{2} B_{2}$. By symmetry it follows that $\varphi_{i}\left(B_{i} C_{i}\right)=C_{i+1} B_{i+1}$ for all $i \in$ $\{1,2,3\}$. Now, let $\Delta^{\prime}$ be the group defined in Equation (3.5). Clearly, $\Delta \leq \Delta^{\prime}$ by the definition of $\varphi_{i}$ for $i \in\{1,2,3\}$. So let $\left(g_{1}^{\prime}, g_{2}^{\prime}, g_{3}^{\prime}\right) \in \Delta^{\prime}$. Since $\Delta$ is subdirect there is a $\left(g_{1}^{\prime}, g_{2}, g_{3}\right) \in \Delta$ with $g_{2} B_{2}=g_{2}^{\prime} B_{2}$. So we can assume that $g_{2}=g_{2}^{\prime}$. But then, by 2-factor injectivity of $\Delta^{\prime}$, we get that $g_{3}=g_{3}^{\prime}$.

Finally for $\left(g_{1}, g_{2}, g_{3}\right) \in \Delta$ we have that

$$
\varphi_{i}\left(g_{i} B_{i} C_{i}\right)=\varphi_{i}\left(g_{i} C_{i}\right) \varphi_{i}\left(B_{i} C_{i}\right)=g_{i+1} C_{i+1} B_{i+1}=g_{i+1} B_{i+1} C_{i+1} .
$$

So

$$
\varphi_{2}\left(\varphi_{1}\left(g_{1} B_{1} C_{1}\right)\right)=\varphi_{3}^{-1}\left(g_{1} B_{1} C_{1}\right) \quad \text { for all } g_{1} \in G_{1}
$$


THEOREM 3.15. There is a natural one-to-one correspondence between degenerate 2-factor injective subdirect products of the product $G_{1} \times G_{2} \times G_{3}$ and the tuples of the form $\left(B_{1}, B_{2}, B_{3}, C_{1}, C_{2}, C_{3}, \varphi_{1}, \varphi_{2}, \varphi_{3}\right)$ for which for all $i \in\{1,2,3\}$ (indices taken modulo 3) we have

(1) $B_{i}, C_{i} \unlhd G_{i}$,

(2) $B_{i} \cap C_{i}=1$,

(3) $\left[B_{i}, C_{i}\right]=1$,

(4) $G_{i} / C_{i} \stackrel{\varphi_{i}}{\cong} G_{i+1} / B_{i+1}$,

(5) $\varphi_{i}\left(B_{i} C_{i}\right)=C_{i+1} B_{i+1}$,

(6) $\varphi_{3}\left(\varphi_{2}\left(\varphi_{1}\left(g_{1} B_{1} C_{1}\right)\right)\right)=g_{1} B_{1} C_{1}$ for all $g_{1} \in G_{1}$.

P r o of. The statement follows from Theorem 1.1 Lemma 3.13 and 3.14

By combining Theorem 3.15 with Lemma 3.10 we obtain the following correspondence result for degenerate subdirect products.

CoRollaRY 3.16. There is a natural one-to-one correspondence between degenerate subdirect products of $G_{1} \times G_{2} \times G_{3}$ and the tuples that are of the form $\left(N_{1}, N_{2}, N_{3}, B_{1}, B_{2}, B_{3}, C_{1}, C_{2}, C_{3}, \varphi_{1}, \varphi_{2}, \varphi_{3}\right)$ for which for all $i \in\{1,2,3\}$ (indices taken modulo 3) we have

(1) $N_{i} \unlhd G_{i}$,

(2) $B_{i}, C_{i} \unlhd G_{i} / N_{i}$,

(3) $B_{i} \cap C_{i}=1$,

(4) $\left[B_{i}, C_{i}\right]=1$,

(5) $\left(G_{i} / N_{i}\right) / C_{i} \stackrel{\varphi_{i}}{\cong}\left(G_{i+1} / N_{i+1}\right) / B_{i+1}$,

(6) $\varphi_{i}\left(B_{i} C_{i}\right)=C_{i+1} B_{i+1}$,

(7) $\varphi_{2}\left(\varphi_{1}\left(g_{1} B_{1} C_{1}\right)\right)=\varphi_{3}^{-1}\left(g_{1} B_{1} C_{1}\right)$ for all $g_{1} \in G_{1} / N_{1}$.

We conclude with the particular case in which $\pi_{i}(\Delta)$ has a complement in $G_{i}$ for some $i \in\{1,2,3\}$. Example 3.4 described in the previous section is of this form. For this case we only obtain an injection to tuples, rather than a one-to-one correspondence. We will exploit having this injection in the next section for small special cases in our analysis of subdirect products of symmetric groups.

THEOREM 3.17. Suppose $G_{1}=E_{1} \rtimes K$ is a semidirect product. There is an injective mapping from the set of 2-factor injective subdirect products $\Delta$ of $G_{1} \times$ $G_{2} \times G_{3}$ with $\pi_{1}(H)=E_{1}$ and $B_{1}=C_{1}$ to the tuples $(\kappa, \iota)$, where $G_{2} \stackrel{\kappa}{\cong} G_{3}$ and $\iota$ is an automorphism of $G_{1}$ that fixes $K$ as a set. Moreover, if $(\kappa, \iota)$ is in the 


\section{SUBGROUPS OF 3-FACTOR DIRECT PRODUCTS}

image of this mapping, then $\left(\kappa, \iota^{\prime}\right)$ is also in the image for every automorphism $\iota^{\prime}$ of $G_{1}$ that fixes $K$ as a set.

P r o of. Let $\Delta$ be a 2 -factor injective subdirect product of $G_{1} \times G_{2} \times G_{3}$ satisfying $\pi_{1}(H)=E_{1}$ and $B_{1}=C_{1}$.

For every element $g_{2} \in G_{2}$ there is exactly one element $\left(k_{1}, g_{2}, g_{3}\right) \in \Delta$ with $k_{1} \in K$. We obtain a well defined isomorphism $\kappa$ from $G_{2}$ to $G_{3}$.

Suppose now that $\Delta^{\prime}$ is a second 2-factor injective subdirect product $G_{1} \times$ $G_{2} \times G_{3}$ satisfying $\pi_{1}(H)=E_{1}$ and $B_{1}=C_{1}$ for which we obtain the same isomorphism $\kappa$. Then we can construct an automorphism $\iota$ of $G_{1}$ as follows.

For every $g_{2} \in G_{2}$ there is exactly one $\left(k, g_{2}, \kappa\left(g_{2}\right)\right) \in \Delta$. There is also an element of the form $\left(k^{\prime}, g_{2}, \kappa\left(g_{2}\right)\right) \in \Delta^{\prime}$. We define $\iota_{K}: K \rightarrow K$ so that it maps $k$ to $k^{\prime}$, this gives us an automorphism $\iota_{K}$ of $K$. For every $e \in E_{1}$ there is an element $\left(e, g_{2}, 1\right) \in \Delta$. There is also an element $\left(e^{\prime}, g_{2}, 1\right) \in \Delta^{\prime}$ and define the map $\iota_{E}: E_{1} \rightarrow E_{1}$ by mapping $e$ to $e^{\prime}$. Then the map $\iota_{E}$ is an automorphism of $E$.

We claim that the map that sends $e \cdot k$ to $\iota_{E}(e) \cdot \iota_{K}(k)$ is an automorphism of $G_{1}$. To see this suppose $a=\left(e_{1}, h, 1\right)(k, g, \kappa(g))$ and $\bar{a}=\left(\overline{e_{1}}, \bar{h}, 1\right)(\bar{k}, \bar{g}, \kappa(\bar{g}))$ are two elements in $\Delta$. Then $\iota(a)=\left(\iota_{E}\left(e_{1}\right), h, 1\right)\left(\iota_{K}(k), g, \kappa(g)\right)$ and $\iota(\bar{a})=$ $\left(\iota_{E}\left(\overline{e_{1}}\right), \bar{h}, 1\right)\left(\iota_{K}(\bar{k}), \bar{g}, \kappa(\bar{g})\right)$ are elements of $\Delta^{\prime}$.

For the products we obtain that

$$
a \bar{a}=\left(e_{1}{\overline{e_{1}}}^{k}, h \bar{h}^{g}, 1\right)(k \bar{k}, g \bar{g}, \kappa(g) \kappa(\bar{g}))
$$

and

$$
\iota(a) \iota(\bar{a})=\left(\iota_{E}\left(e_{1}\right) \iota_{E}\left(\overline{e_{1}}\right)^{\iota_{K}(k)}, h \bar{h}^{g}, 1\right)\left(\iota_{K}(k) \overline{\iota_{K}(k)}, g \bar{g}, \kappa(g) \kappa(\bar{g})\right) .
$$

To conclude that $\iota$ is an isomorphism we now only need to argue that $\iota_{E}\left(\overline{e_{1}}\right)^{\iota_{K}}(k)$ is equal to $\iota_{E}\left({\overline{e_{1}}}^{k}\right)$. However, this is the case since $\left({\overline{e_{1}}}^{k}, h^{g}, 1\right) \in \Delta$ as well as $\left(\iota_{E}\left(e_{1}\right)^{\iota_{K}(k)}, h^{g}, 1\right) \in \Delta^{\prime}$.

Now suppose that $\Delta$ is a subdirect product with $\pi_{1}(H)=B_{1}=C_{1}$ and let $\kappa: G_{2} \rightarrow G_{3}$ be defined as above. Let $\iota$ be an automorphism of $G_{1}$ that fixes $K$, then $\Delta^{\prime}=\left\{\left(\iota\left(g_{1}\right), g_{2}, g_{3}\right) \mid\left(g_{1}, g_{2}, g_{3}\right) \in \Delta\right\}$ is a subdirect product of $G_{1} \times G_{2} \times G_{3}$. If we apply the above construction for the automorphism of $G_{1}$, we reobtain $\iota$. This shows that the construction of $\iota$ from $\Delta^{\prime}$ and the construction of $\Delta^{\prime}$ from $\iota$ are inverses to one another.

Note that in the theorem, the isomorphism $\kappa$ associated with a subdirect product is canonical (it only depends on the choice of $K$ ) but the choice of $\iota$ is not. 


\section{Subdirect products of two or three symmetric groups}

In this section we apply the correspondence theorems to count the subdirect products of the direct product of three symmetric groups. We first reduce this problem to counting the number of 2-factor injective subdirect products. For finite groups $G_{1}, \ldots, G_{k}$ let $\ell\left(G_{1}, \ldots, G_{k}\right)$ be the number of subdirect products of $G_{1} \times \cdots \times G_{k}$. Furthermore, for $k=3$, we denote by $\ell_{2 \text {-inj }}\left(G_{1}, \ldots, G_{3}\right)$ the number of 2-factor injective subdirect products.

LemmA 4.1. Let $G_{1}, G_{2}, G_{3}$ be finite non-trivial groups. Then

$$
\begin{aligned}
\ell\left(G_{1}, G_{2}, G_{3}\right)= & \sum_{N_{i} \unlhd G_{i}} \ell_{2-i n j}\left(G_{1} / N_{1}, G_{2} / N_{2}, G_{3} / N_{3}\right) \\
= & \ell\left(G_{1}, G_{2}\right)+\ell\left(G_{2}, G_{3}\right)+\ell\left(G_{1}, G_{3}\right)-2 \\
& +\sum_{N_{i} \triangleleft G_{i}} \ell_{2-i n j}\left(G_{1} / N_{1}, G_{2} / N_{2}, G_{3} / N_{3}\right) .
\end{aligned}
$$

P r o o f. The first equality follows from the correspondence described in Lemma 3.10. The second equality follows by noting that the direct product is counted three times, so 2 has to be subtracted.

We are interested in the number $\ell\left(n_{1}, n_{2}, n_{3}\right):=\ell\left(S_{n_{1}}, S_{n_{2}}, S_{n_{3}}\right)$, where $S_{n_{i}}$ is the symmetric group of a set with $n_{i}$ elements. Recall, that every factor group of a symmetric group is isomorphic to a symmetric group over another set. Thus, by the previous lemma, it suffices to compute the numbers $\ell\left(n_{1}, n_{2}\right)$ and $\ell_{2 \text {-inj }}\left(n_{1}, n_{2}, n_{3}\right):=\ell_{2 \text {-inj }}\left(S_{n_{1}}, S_{n_{2}}, S_{n_{3}}\right)$.

We start by analyzing the situation for two factors.

LEMMA 4.2. Let $n_{1}, n_{2} \geq 2$. For the number $\ell\left(n_{1}, n_{2}\right)$ of subdirect products of $S_{n_{1}} \times S_{n_{2}}$ we have

$$
\ell\left(n_{1}, n_{2}\right)= \begin{cases}2 & \text { if } n_{1} \neq n_{2} \text { and }\left\{n_{1}, n_{2}\right\} \neq\{3,4\}, \\ 8 & \text { if }\left\{n_{1}, n_{2}\right\}=\{3,4\} \\ n_{1} !+2 & \text { if } n_{1}=n_{2} \notin\{2,4,6\} \\ 2 & \text { if } n_{1}=n_{2}=2, \\ n_{1} !+8 & \text { if } n_{1}=n_{2}=4 \\ 2 n_{1} !+2 & \text { if } n_{1}=n_{2}=6 .\end{cases}
$$

P r o of. We assume for our considerations that $n_{1} \geq n_{2}$. Let $\left(S_{n_{1}}, S_{n_{2}}, N_{1}, N_{2}, \varphi\right)$ be a tuple corresponding to a subdirect product via the correspondence of Theorem 2.2. 


\section{SUBGROUPS OF 3-FACTOR DIRECT PRODUCTS}

- If $N_{1}=1$, then $N_{2}=1$ and $n_{1}=n_{2}$. The number of isomorphisms from $S_{n_{1}}$ to $S_{n_{1}}$ is

$$
i\left(n_{1}\right)= \begin{cases}1 & \text { if } n_{1}=2 \\ 2 n ! & \text { if } n_{1}=6 \\ n ! & \text { otherwise }\end{cases}
$$

This corresponds to the number of possible choices for $\varphi$. (These are the diagonal subgroups.)

- If $N_{1}=S_{n_{1}}$, then $N_{2}=S_{n_{2}}$. There is only one subgroup of this type. (This is the direct product).

- If $N_{1}=A_{n_{1}}\left(n_{1} \geq 3\right)$, then $N_{2}=A_{n_{2}}$, since the only index 2 subgroup that a symmetric group can have is the alternating group. There is only one subgroup of this type.

If $n_{1} \neq 4$, then $N_{1} \in\left\{1, A_{n_{1}}, S_{n_{1}}\right\}$, and we already considered all these cases. Suppose now that $n_{1}=4$ and $n_{2} \leq 4$. Then $N_{1} \in\left\{1, V, A_{n_{1}}, S_{n_{1}}\right\}$, where $V$ is the Klein-four-group. Three of the cases are considered above.

- If $N_{1}=V$, then $N_{2}=V$ and $n_{2}=4$ or $N_{2}=1$ and $n_{2}=3$. In either case there are 6 options for $\varphi$.

In the following we use "\{\{" and "\}\}" to denote multisets.

LEMMA 4.3. Let $n_{1}, n_{2}, n_{3} \geq 2$. For the number $\ell_{2 \text {-inj }}\left(n_{1}, n_{2}, n_{3}\right)$ of 2-factor injective subdirect products of $S_{n_{1}} \times S_{n_{2}} \times S_{n_{3}}$ we have

$$
\ell_{2-i n j}\left(n_{1}, n_{2}, n_{3}\right)= \begin{cases}\left(n_{1} !\right)^{2} & \text { if } n_{1}=n_{2}=n_{3} \notin\{2,3,4,6\}, \\ 2 & \text { if } n_{1}=n_{2}=n_{3}=2, \\ \left(n_{1} !\right)^{2}+2 n_{1} ! & \text { if } n_{1}=n_{2}=n_{3}=3, \\ \left(n_{1} !\right)^{2}+6 n_{1} ! & \text { if } n_{1}=n_{2}=n_{3}=4, \\ \left(2 n_{1} !\right)^{2} & \text { if } n_{1}=n_{2}=n_{3}=6, \\ 1440 & \text { if }\left\{\left\{n_{1}, n_{2}, n_{3}\right\}=\{\{2,6,6\},\right. \\ n ! & \text { if }\left\{\left\{n_{1}, n_{2}, n_{3}\right\}=\{\{2, n, n\}, \text { for } n \notin\{2,6\},\right. \\ 144 & \text { if }\left\{\left\{n_{1}, n_{2}, n_{3}\right\}=\{\{3,4,4\},\right. \\ 0, & \text { otherwise. }\end{cases}
$$

P r o of. Suppose without loss of generality that $n_{1} \geq n_{2} \geq n_{3}$. Let $\Delta \leq$ $S_{n_{1}} \times S_{n_{2}} \times S_{n_{3}}$ be a 2-factor injective subdirect product. Define $H_{1}, H_{2}, H_{3}, H$ as in Section 3.2. Let $E_{i}=\pi_{i}(H)$. Then $H \unlhd \Delta$ and $E_{i} \unlhd S_{n_{i}}$ for $i \in\{1,2,3\}$. By Theorem 1.1] it holds that $S_{n_{1}} / E_{1} \cong S_{n_{2}} / E_{2} \cong S_{n_{3}} / E_{3}$. Also, by Theorem 


\section{DANIEL NEUEN-PASCAL SCHWEITZER}

3.11 for $H$ we get a canonical tuple $\left(B_{1}, B_{2}, B_{3}, C_{1}, C_{2}, C_{3}, \varphi_{1}, \varphi_{2}, \varphi_{3}\right)$ with subgroups $B_{i}, C_{i} \unlhd E_{i}$, such that $B_{i} \cap C_{i}$ is Abelian and $B_{i} C_{i}=E_{i}$. We obtain the following options.

- If $E_{1}=1$, then $n_{1}=n_{2}=n_{3}$ and $E_{2}=E_{3}=1$. We have $B_{i}=C_{i}=1$ for $i \in\{1,2,3\}$. In this case $H=1$ and $\Delta$ is degenerate. By Theorem 3.15 there are $i\left(n_{1}\right)^{2}$ groups of this type, where $i\left(n_{1}\right)$ is the number of isomorphisms from $S_{n_{1}}$ to $S_{n_{1}}$. This corresponds to the choices for $\varphi_{1}$ and $\varphi_{2}$. For $\varphi_{3}$ we get $\varphi_{3}^{-1}=\varphi_{1} \circ \varphi_{2}$.

- If $E_{1}=V$, then $n_{1}=4$ and $S_{n_{1}} / E_{1} \cong S_{3}$. In this case $\left\{n_{1}, n_{2}, n_{3}\right\} \subseteq\{3,4\}$. Let us first consider the case that $n_{3}=3$. Then $B_{3}=C_{3}=E_{3}=1$ and we can thus apply Theorem 3.15. By Lemma 3.9 we conclude that $C_{1}=B_{2}=1$ and $E_{1}=B_{1}=C_{2}=V$. Using the correspondence given in Theorem 3.15 the number of such groups equals the number of pairs $\left(\varphi_{1}, \varphi_{2}\right)$, where $\varphi_{1}$ is an isomorphism from $S_{4}$ to $S_{4}$ and $\varphi_{2}$ is an isomorphism from $S_{3}$ to $S_{3}$. There are 144 such pairs.

Next let us consider the case $n_{1}=n_{2}=n_{3}=4$. This implies that $B_{i}=$ $C_{i}=E_{i}=V$ for all $i \in\{1,2,3\}$. Since $S_{4}$ is the semidirect product $V \rtimes S_{3}$, we can apply Theorem 3.17. For every isomorphism $\kappa$ from $S_{n_{2}}=S_{4}$ to $S_{n_{3}}=S_{4}$ we can find a subdirect product realizing $\kappa$ by setting $\Delta=$ $\left\langle\left\{(k, g, \kappa(g)) \mid g \in S_{4}, k \in S_{3} \cap g V\right\} \cup\left\{\left(a, a^{-1}, 1\right) \mid a \in V\right\}\right\rangle$. Thus, by Theorem 3.17 the number of such subdirect products is equal to the number of pairs $(\kappa, \iota)$, where $\kappa$ is an isomorphism from $S_{4}$ to $S_{4}$ and $\iota$ is an automorphism of $S_{4}$ that fixes $V$ as a set. There are $6 n_{1} !=144 \mathrm{such}$ pairs.

- If $E_{1}=A_{n_{1}}$ (and $n_{1} \geq 3$ ), then $E_{2}=A_{n_{2}}$ and $E_{3}=A_{n_{3}}$. By applying Theorem 3.11 to $H$ it follows that either $n_{1}=n_{2}=n_{3}=3$ or $n_{1}=n_{2}>$ $n_{3}=2$. In the former case $B_{i}=C_{i}=A_{3}=\mathbb{Z}_{3}$ for $i \in\{1,2,3\}$ and in total there are $2 n_{1} !=12$ groups of this type by Theorem 3.17 (by the same arguments as in the previous case). In the latter case $B_{1}=C_{2}=A_{n_{1}}$ and $C_{1}=B_{2}=B_{3}=C_{3}=1$. So $\Delta$ is degenerate and by Theorem 3.15 there are in total $i\left(n_{1}\right) \cdot i(2)=i\left(n_{1}\right)$ groups of this type, where again $i\left(n_{1}\right)$ is the number of isomorphisms from $S_{n_{1}}$ to $S_{n_{1}}$.

- If $E_{1}=S_{n_{1}}$, then $E_{2}=S_{n_{2}}$ and $E_{3}=S_{n_{3}}$. In this case $H=\Delta$. Using the correspondence described in Theorem 3.11 we get that $n_{1}=n_{2}=n_{3}=2$ and $B_{i}=C_{i}=S_{2}$ for $i \in\{1,2,3\}$. Since there is only one isomorphism from $S_{2}$ to $S_{2}$ there is exactly one option in this case, namely the group given in Example 3.2 for $G_{1}=S_{2}$.

Proof of Theorem 1.2. Using Lemma 4.1 and 4.3 we get

$$
\ell\left(n_{1}, n_{2}, n_{3}\right)=\sum_{i<j} \ell\left(n_{i}, n_{j}\right)+
$$




\section{SUBGROUPS OF 3-FACTOR DIRECT PRODUCTS}

$$
\begin{cases}\ell_{2 \text {-inj }}\left(n_{1}, n_{1}, n_{1}\right)+3 \ell_{2 \text {-inj }}\left(2, n_{1}, n_{1}\right) & \text { if } n_{1}=n_{2}=n_{3}, \\ \ell_{2 \text {-inj }}(2,4,4)+\ell_{2 \text {-inj }}(2,3,3) & \text { if } n_{2}=n_{3}=4, \\ \ell_{2 \text {-inj }}(2,3,3) & \text { if } n_{2} \in\{3,4\}, n_{3}=3, \\ \ell_{2 \text {-inj }}\left(2, m_{1}, m_{1}\right) & \text { if }\left\{n_{1}, n_{2}, n_{3}\right\}=\left\{\left\{m_{1}, m_{1}, m_{2}\right\}\right\} \\ & \text { for } m_{1} \neq m_{2}, m_{1} \geq 5, \\ 0, & \text { otherwise. }\end{cases}
$$

Then apply Lemmas 4.2 and 4.3 .

Recall that the finitely many cases not covered by the Theorem are listed in Table 1. These numbers were calculated using Lemmas 4.1 4.2 and 4.3. However, these numbers were also double-checked with the computer algebra system GAP [6].

\section{REFERENCES}

[1] ANDERSON, D. D.-CAMILLO, V.: Subgroups of direct products of groups, ideals and subrings of direct products of rings, and Goursat's lemma. In: Rings, Modules and Representations. In: Contemp. Math. Vol. 480, Amer. Math. Soc., Providence, RI, 2009, pp 1-12; http://dx.doi.org/10.1090/conm/480/09364 doi:10.1090/conm/480/09364

[2] BAUER, K.-SEN, D. -ZVENGROWSKI, P.: A generalized Goursat lemma, Tatra Mt. Math. Publ. textbf64 (2015) 1-19; doi:10.1515/tmmp-2015-0039

[3] BRIDSON, M. R.-HOWIE, J.-MILLER, C. F. III,-SHORT, H.: On the finite presentation of subdirect products and the nature of residually free groups, Amer. J. Math. 135 (2013), no. 4, 891-933;

http://dx.doi.org/10.1353/ajm.2013.0036 doi:10.1353/ajm.2013.0036

[4] BRIDSON, M. R.-MILLER, C. F. III.: Structure and finiteness properties of subdirect products of groups. Proc. Lond. Math. Soc. 98 (2009) no. 3, 631-651; http://dx.doi.org/10.1112/plms/pdn039 doi:10.1112/plms/pdn039

[5] BUTLER, L. M.: Subgroup lattices and symmetric functions. Mem. Amer. Math. Soc. 112 (1994), no. 539, pp. vi-160; http://dx.doi.org/10.1090/memo/0539 doi:10.1090/memo/0539

[6] THE GAP GROUP: GAP - Groups, Algorithms, and Programming, Version 4.8.3, 2016; http://www.gap-system.org

[7] GEKAS, S.: Deciphering subgroups of direct products; ArXiv e-prints, Septembr 2015. arXiv:1509.03245.

[8] GOURSAT, E.: Sur les substitutions orthogonales et les divisions régulières de l'espace. Ann. Sci. École Norm. Sup. (3), (1889) 9-102; http://www.numdam.org/item?id=ASENS_1889_3_6__9_0

[9] HALL, M. JR.: The theory of groups. Chelsea Publishing Co., New York, 1976. (Reprinting of the 1968 edition) 


\section{DANIEL NEUEN-PASCAL SCHWEITZER}

[10] HAMPEJS, M.-TÓTH, L.: On the subgroups of finite abelian groups of rank three. Ann. Univ. Sci. Budapest. Sect. Comput. 39 (2013), 111-124.

[11] LANG, S.: Algebra (3rd ed.) In:Graduate Texts in Mathematics Vol. 211, Springer-Verlag, New York, 2002. http://dx.doi.org/10.1007/978-1-4613-0041-0, doi:10.1007/978-1-4613-0041-0

[12] MILLER, M. D.: On the lattice of normal subgroups of a direct product, Pacific J. Math. 60 (1975) no. 2, 153-158.

[13] PETRILLO, J.: Counting subgroups in a direct product of finite cyclic groups. College Math. J. textbf42 (2011), no. 3, 215-222. http://dx.doi.org/10.4169/college.math.j.42.3.215 doi:10.4169/college.math.j. 42.3 .215 .

[14] REMAK, R.: Über Untergruppen direkter Produkte von drei Faktoren. J. Reine Angew. Math. 166 (1932), 65-100. http://dx.doi.org/10.1515/crll.1932.166.65 doi:10.1515/crll.1932.166.65

[15] TÓTH, L.: Subgroups of finite abelian groups having rank two via Goursat's lemma. Tatra Mt. Math. Publ. 59 (2014), 93-103; doi:10.2478/tmmp-2014-0021

[16] TĂRNĂUCEANU, M.: An arithmetic method of counting the subgroups of a finite abelian group. Bull. Math. Soc. Sci. Math. Roumanie (N.S.), 53(101) (2010), no. 4, 373-386.

Received July 25, 2018

\author{
Daniel Neuen \\ Department of Computer Science 7 \\ RWTH Aachen University \\ Ahornstraße 55 \\ 52074 Aachen \\ GERMANY
}

E-mail: neuen@informatik.rwth-aachen.de

Pascal Schweitzer

Department of Computer Science

Technical University Kaiserslautern

Department of Computer Science

Postfach 3049

67663 Kaiserslautern

GERMANY

E-mail: schweitzer@cs.uni-kl.de 